This item was submitted to Loughborough's Research Repository by the author.

Items in Figshare are protected by copyright, with all rights reserved, unless otherwise indicated.

\title{
An efficient algorithm for anomaly detection in a flight system using dynamic Bayesian networks
}

\author{
PLEASE CITE THE PUBLISHED VERSION
}

https://doi.org/10.1007/978-3-642-34487-9_75

\section{PUBLISHER}

Springer

VERSION

AM (Accepted Manuscript)

\section{PUBLISHER STATEMENT}

This version of the contribution has been accepted for publication, after peer review (when applicable) but is not the Version of Record and does not reflect post-acceptance improvements, or any corrections. The Version of Record is available online at: https://doi.org/10.1007/978-3-642-34487-9_75. Use of this Accepted Version is subject to the publisher's Accepted Manuscript terms of use https://www.springernature.com/gp/open-research/policies/accepted-manuscript-terms

\section{LICENCE}

\section{All Rights Reserved}

\section{REPOSITORY RECORD}

Saada, Mohamad, and Qinggang Meng. 2012. "An Efficient Algorithm for Anomaly Detection in a Flight System Using Dynamic Bayesian Networks". Loughborough University. https://hdl.handle.net/2134/18319910.v1. 


\title{
An Efficient Algorithm for Anomaly Detection in a Flight System using Dynamic Bayesian Networks
}

\author{
Mohamad Saada and Qinggang Meng \\ Department of Computer Science \\ Loughborough University \\ Loughborough \\ United Kingdom \\ M.Saada@lboro.ac.uk \\ Q.Meng@lboro.ac.uk
}

\begin{abstract}
Despite the fact that Dynamic Bayesian Network models have become a popular modelling platform to many researchers in recent years, not many have ventured into the realms of data anomaly and its implications on DBN models. An abnormal change in the value of a hidden state of a DBN will cause a ripple-like effect on all descendent states in current and consecutive slices. Such a change could affect the outcomes expected of such models. In this paper we propose a method that will detect anomalous data of past states using a trained network and data of the current network slice. We will build a model of pilot actions during a flight, this model is trained using simulator data of similar flights. Then our algorithm is implemented to detect pilot errors in the past given only current actions and instruments data.
\end{abstract}

Keywords: Anomaly Detection, Dynamic Bayesian Networks, Intelligent Systems, Machine Learning

\section{Introduction}

Data anomalies can occur due to many different reasons, it can be due to a sensor reading error, or a communication error while data is being transmitted through a network, it can be a new type of a network attack which has not been encountered before, or it can simply be due to unexpected and rare system behaviour. The list goes on but in all cases data anomalies share the property of being a type of data that is deviated away from normal data patterns. Anomaly Detection is a very helpful instrument wherever it is applied, banks detect suspicious invalid transactions through the use of anomaly detection, it helps maintain a good level of Quality of Service in network communications, or detect a failing components of a mechanical system. Anomaly detection is useful in endless scenarios, and that is why it has been implemented and investigated in many ways, one of which is through the use of probabilistic graphical models. Many 
researchers have taken different approaches to solve the anomaly detection problem, but most of these approaches belong to three main streams used to solve the problem of data anomaly detection as shown by $[1,2]$, we list these below:

- Unsupervised Approach: Works by detecting anomalies without having any prior information about them.

- Supervised Approach: Works by modelling both anomalous data and normal data, through manually specifying which data is considered anomalous and which is normal.

- Semi-Supervised Approach: Works by modelling normal data only, and then uses the modelled data in the algorithm that detects anomalous data.

Not many researchers have Dynamic Bayesian Network models as basis for their approach, in [3] researchers have developed two machine learning methods a coupled and uncoupled DBN Anomaly Detector which aim to detect erroneous data in two different windspeeds data streams in real time. These methods can work on single or multiple data streams in real time. And in [4] researchers suggested an anomaly detection algorithm based on the use of a new implementation of the Dirichlet process precision parameter, outlier detection is done by calculating a maximum a posteriori (MAP) of the data partition, where observations forming small or singleton clusters are deemed as anomalies. Researchers in [5] have used a Bayesian Network to model the outliers as an "unlikely events under the current favored theory of the domain", their approach is based on using a Bayesian network modelling the background knowledge coupled with two rules to detect the outliers, their approach not only focuses on detecting outliers but also on explaining why these data are considered outliers. Researchers in [6] use an unsupervised approach towards detecting fraud operations in a stock exchange market, they use Peer Group Analysis (PGA) technique which is concerned with characterizing the expected pattern of behaviour around the targeted time series financial sequence in terms of the behaviour of similar objects and then detect outliers through the detection of difference in evolution of the actual behaviour and expected behaviour.

When using probabilistic models such as Dynamic Bayesian Networks to model a certain environment or a system, modellers usually work with large amounts of data, and that is due to the fact that the main reason behind probabilistic modelling is to extract useful information about the environment from its data, which otherwise cannot be easily extracted or interpreted, not even by experts. And as we know wherever there is manipulation of large amounts of data there are bound to be data anomalies which can have damaging effects on the processes manipulating this data which could lead to erroneous outcome. And these data anomalies can affect the information that is gathered from the model greatly, and this is why in this paper we are focusing on the detection of data anomalies that occur while working with Dynamic Bayesian Networks which will be denoted from here on as DBN. 


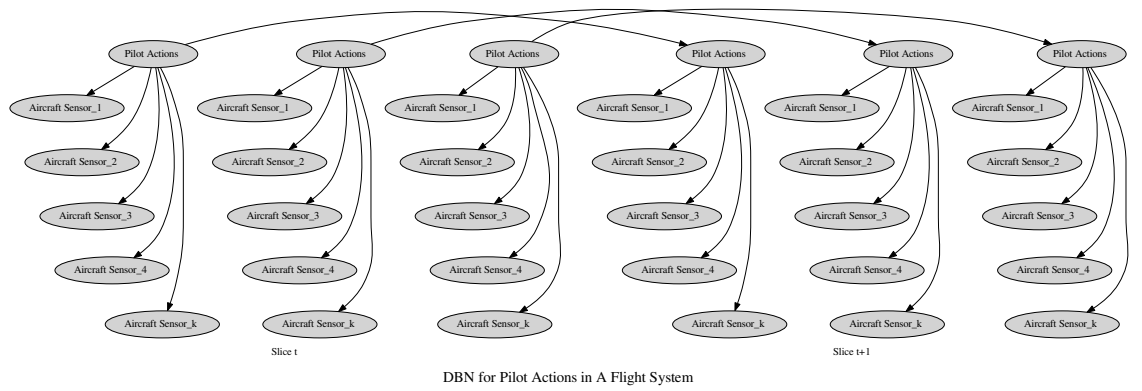

Fig. 1: A Simplified DBN Model for Pilot Actions in a Flight System

\section{Dynamic Bayesian Network Model}

A Dynamic Bayesian Network is the extension of Bayesian Networks to model probability distributions of sets of random variables over time [7]. Bayesian Network on the other hand are a type of probabilistic models that are based on directed acyclic graphs (DAGs) [8], the nodes in this model represent propositional variables of interest and the links between them represent the dependencies among these variables [8], and these dependencies are quantified by conditional probabilities of each node given its parents in the network. Nodes in our DBN model $Z_{t}^{k}$ are divided into two sets where $t$ represents the slice number which indicates the time variable, and $k$ is the number of nodes in each slice. The first set contains the hidden state nodes $X_{t}^{n}=\left\{X_{t}^{1}, X_{t}^{2}, X_{t}^{3}, \ldots, X_{t}^{n}\right\}$, where $n$ represents the number of hidden states in each slice. Hidden states represent immeasurable variables in our model, and these are usually the variables that we aim to gather information about. And the second set is the set of observable nodes $Y_{t}^{m}=\left\{Y_{t}^{1}, Y_{t}^{2}, Y_{t}^{3}, \ldots, Y_{t}^{m}\right\}$, where $m$ represents the number of observable nodes in each slice. Observable nodes represent variables that can be measured and are completely or partially observable. These are sometimes called evidence nodes. Note that $n+m=k$ in our model.

Each DBN slice contains $n$ hidden variable nodes which represent pilot actions, and $k$ observable and measurable nodes which represent different simulation variables, and these are all observable in our model. The connections between model nodes are set according to actual relationships between the modelled environment variables. And inter slice connections are restricted to hidden nodes. As we mention this we should mention that with the DBN model we set a prior probability for the first slice in the network $P\left(X_{1}\right)$, and we have a statetransition function $P\left(X_{t} \mid X_{t-1}\right)$, and an observation function $P\left(Y_{t} \mid X_{t}\right)$. Since we are working with $\mathrm{DBN}$ we assume that the model is first-order Markov [7] (i.e. $P\left(X_{t} \mid X_{1: t-1}\right)=P\left(X_{t} \mid X t-1\right)$ ), and that observations are conditionally first Markov [7] (i.e. $P\left(Y_{t} \mid Y_{t-1}, X_{t}\right)=P\left(Y_{t} \mid X_{t}\right)$ ). So from the two previous 
assumptions we conclude that inter slice relations are limited to hidden states, observable states are only related to their parent states in the same slice.

Now that the model is complete we would like to explain how it is usually used, after the model is built to model different variables in the environment and their relationships, we train the model using the Expectation Maximization algorithm, for the purpose of training we use a number of data sets which usually contain data for all variables (hidden and observable), after training we apply inference techniques to gather the information we need about hidden variables, these techniques [7] include filtering, prediction, classification, control, abduction and smoothing. When performing inference a new data set is used, and this data set contains environment data that we wish to gather information about and this data set usually contains only observable nodes data, and here were anomalies effect can be detected. Our anomaly detection algorithm works using some inference techniques as basic steps to enable it detect data anomalies.

\section{Anomaly Detection in a DBN}

Anomaly detection is the process of detecting patterns in data that do not conform to the expected normal patterns. Anomalies are also referred to as outliers which Hawkins [9] defines as "an observation that deviates so much from other observations as to arouse suspicions that it was generated by a different mechanism". Our approach to anomaly detection is not based on the typical approach which usually focuses on detecting anomalies in general within data of a given model, instead we take another route. When anomalies occur during the prediction or classification process they often have a ripple-like effect on the descendent states in the same slice and consecutive slices. If the anomaly occurs in one slice, its affect is spread to related states in the same slice and to consecutive slices, albeit the effect is shortly lived and soon all values turn back to normal. So the longer the anomaly occurs, the longer and bigger the effect is. In adaptable online learning models if an anomaly continues to occur for a certain period of time, the model will adapt to it and this anomaly will be then considered the norm. During the inference of trained models new data is used, this data could contain some anomalies when compared to the data that was used to train the model. Data could be considered as an anomaly due to its value which does not belong to the range of acceptable values of a given variable. Or it could have a normal value, but it is not normal for this value to occur at that point of time. The second type of anomalies could pass undetected by the experts, and thus effecting descendent states, and if it continued to occur, it could lead to unexpected values when inference is applied to the model. Our algorithm aims to detect this type of data anomaly. During the inference phase, the model is supplied with a data set containing some anomalies. The anomalies are of an acceptable value but do not occur at the expected time, their effect is propagated to related states in the same and succeeding slices. We suppose that we are able to detect these effects on other state/states $Z_{t}$ at slice $t$. So our assumption is that we are processing slice $t$ and that all states $Z_{t}$ of slice $t$ are observable with 


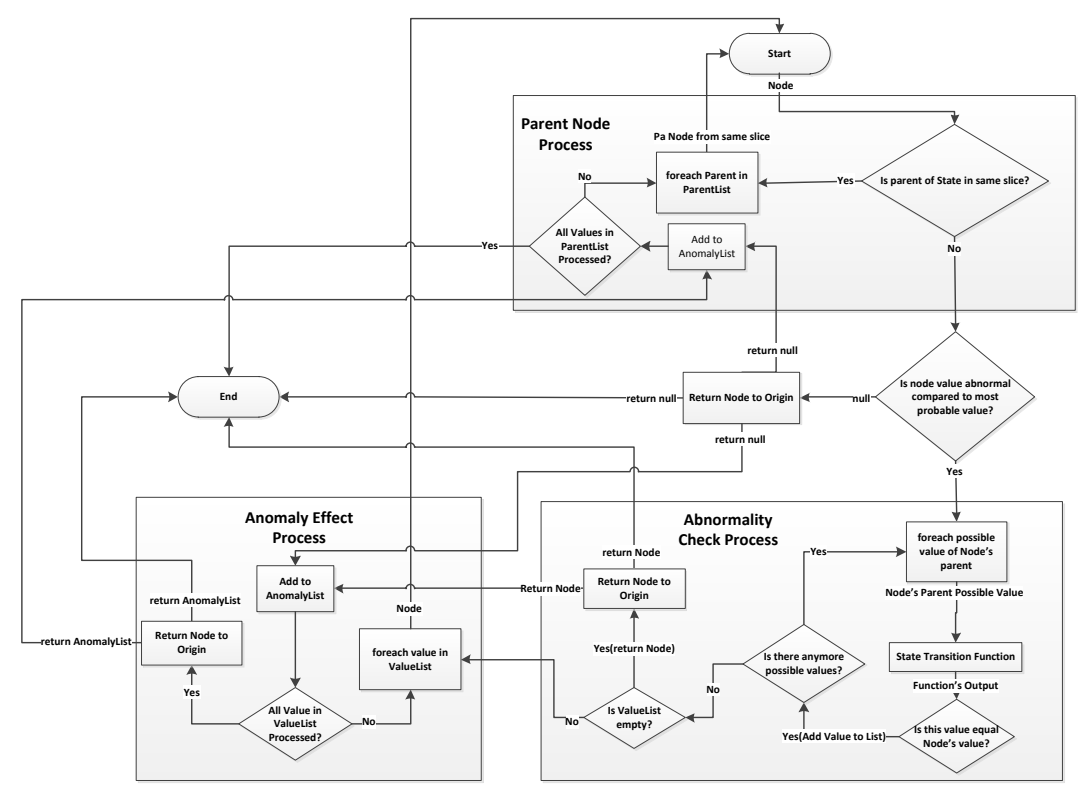

Fig. 2: Flow Chart of Anomaly Detection Algorithm

known state values. Our objective is to go back trough the slices until we can identify in which states $Z_{t-k}$ an anomaly started to occur which have caused the values of future states to be affected and changed. As we mentioned in the previous section, our DBN model is first-order Markov, and observations are conditionally first-order Markov, this leads to the conclusion that hidden states has an affect only on observable states of the same slice and hidden states in the next slice and are only affected by hidden states of the previous slice. We aim to find the node/set of nodes $X_{t-k}^{i}$ in slice $t-k$-where $k$ is unknown- that effectively caused a considerable change of value in state $Z_{t}^{j}$ in slice $t$ in comparison with the data of the trained model.

As it is apparent from algorithm 1, the algorithm takes as input a state $Z_{t}^{j}$ where an abnormal value is detected, and produces as output the state or set of states $X_{t-k}^{i}$ that had an anomaly which caused this abnormal change of value. At first the algorithm makes sure that the input state does not have any parents in the same slice, if parents do exist this means that the state in concern is an evidence state, then the parent/parents -hidden states- of this state is found. In this case the DETECTANOMALY algorithm is re-implemented on the parent node/nodes. The algorithm calculates the highest probability of any expected value of state $Z_{t}^{j}$ at slice $t$ given the trained model. Then this value is compared to probability of the actual value of the state occurring, if there is a large difference between these two values then this data is considered anomalous. 


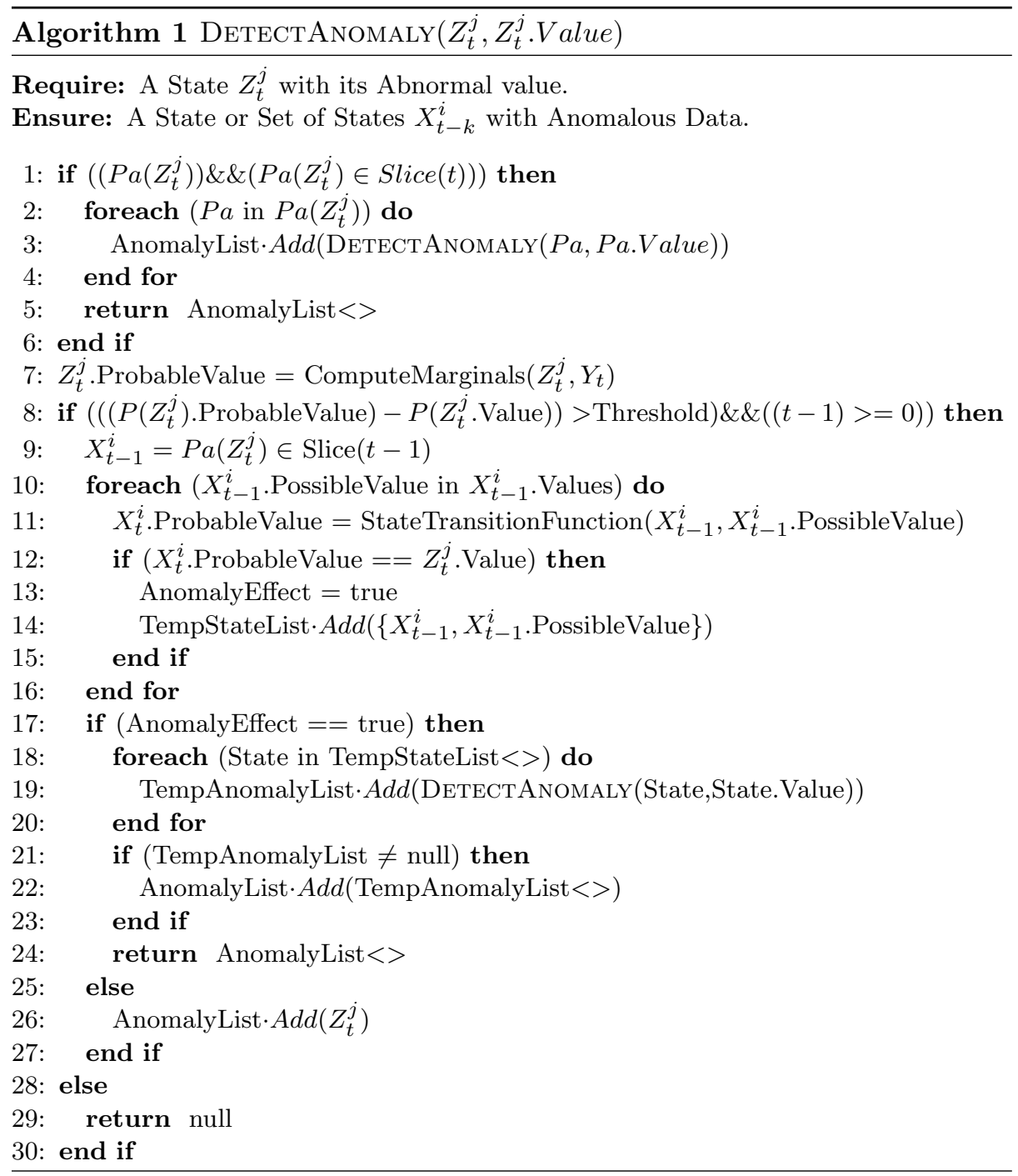

Otherwise the algorithm exits. Next step is to go back one slice and to compute the probability of $Z_{t}^{j}$ occurring with its current values given all possible values for its parent state and the trained model, this is calculated through the state transition function of the DBN model. If there is a value that supports such transition then state $Z_{t}^{j}$ is labelled an affected state, otherwise it is considered as an anomalous state. This process is repeated for all parent states as long as the difference in probability between probable and possible values is above the threshold. When this difference drops below the threshold, the state in that slice is considered normal, and the descendent state in next slice is considered as the first anomalous state in the anomalous path. 


\section{Experiment and Results}

We started by building a DBN model based on a flight scenario, the flight is routed between London Heathrow and East Midlands airports, the flight duration is $50 \mathrm{~min}$ on average. We have used Microsoft ${ }^{\circledR}$ Flight Simulator X as the basis for our simulator, because it is supplied with an SDK which was used to build our software, and another reason is that the simulator is very realistic and accurate and it can give us over 1100 different data variables in high frame rates. We have built our custom software that interacts with the simulator and records all of the flight data online with the desired frame rates.

For training purposes we have recorded data of 30 flights between the two designated airports, the flight direction is always the same. 24 of the recorded flights were flown normally with small differences between them. While the remaining 6 flights had data anomalies occurring in them. These anomalies basically where different than normal pilot actions, such as keeping landing gear lowered much more than the usual time, or keeping Flaps at certain angle after takeoff, then we programmed the simulator to cause an effect on the related variables when these anomalies continued to occur, such as having a rough and bouncy landing when landing gears were kept extended longer than they should, which resemble realistic scenarios. The DBN model that we have built is a single layer DBN network, which compromise of two types of nodes, Hidden nodes $X_{t}^{n}$ which represent immeasurable pilot actions which are annotated manually into the training data sets, and observable nodes $Y_{t}^{m}$ which represent aircraft instrumentation data recorded by our software. Due to the large number of available Sim variables, we had to narrow down the numbers of variables. We have chosen variables which are essential and related to our experiment (i.e. weather data, gps data, altitude and speed data, landing gear data, flaps data, rudder data and data of all cockpit switches that were used during the flight, etc...).

We train the DBN using the Expectation Maximization algorithm in [7], in our training sets we have introduced three types of errors (Landing Gear error, Flaps error, Excess Speed error), each one occurring twice, and the remaining 24 training sets there were no errors. Each one of the errors we have introduced has its own effect. Our algorithm starts working on the slice where the effect appears rather than the slice where the error begins.

In the testing phase we record 9 new data sets with the same types of errors we have introduced, with each error type occurring in 3 different data sets. Note that these data sets do not contain annotated pilot actions, therefore when the algorithm begins data of the observable variables are fetched from the testing data set, whilst data of unobservable variables are entered manually through an annotation step done before running the algorithm.

We start by training the network on 4 training sets, 3 are of normal type and 1 containing an error (Landing Gear error), we run the algorithm on the first of three (Landing Gear error) testing data sets. The algorithm detects $80.7 \%$ of the anomaly list after first run, we increase the number of training sets to 9 sets, 8 of which are normal and 1 containing an error, the algorithm detects $82.6 \%$ of the anomaly list. We continue adding normal training sets to the training step 
and the results increase slightly until algorithm reaches $89.4 \%$ detection of the complete anomaly list. We repeat the same process but using two different error data sets rather than one. A surprising result was that the algorithm came out with a low detection result (around 57\%) when we ran 4 normal data sets with 2 error data sets, we found that the reason for this happing was that we have set a very low value threshold and that the number of error data sets was considered large compared to the number of normal data sets. We have fixed this through increasing the threshold for big error to normal ratios and then increase the ratio of normal to error training sets. After using all 24 normal training sets and 2 error sets we have reached an anomaly list detection rate of $91.1 \%$. Now we do

Table 1: Error Testing Results

\begin{tabular}{cccc}
\hline \hline Learnt DS Lnd Gr Err (1-2) & Flp err(1-2) & exs spd err(1-2) \\
\hline 4 & $80.1 \%-82.7 \%$ & $75.1 \%-77.7 \%$ & $80.9 \%-84.6 \%$ \\
9 & $82.9 \%-84.9 \%$ & $77.9 \%-79.9 \%$ & $82.7 \%-86.4 \%$ \\
14 & $84.2 \%-86.4 \%$ & $79.2 \%-82.6 \%$ & $85.9 \%-88.8 \%$ \\
19 & $86.6 \%-88.5 \%$ & $83.0 \%-84.5 \%$ & $88.8 \%-91.2 \%$ \\
24 & $88.2 \%-90.8 \%$ & $85.2 \%-87.3 \%$ & $91.1 \%-93.6 \%$ \\
\hline
\end{tabular}

the same experiment again but on (Landing Gear error) testing data sets 2 and 3 separately. We get final results of $88.7 \%$ and $90.8 \%$ respectively. We find that the combined accuracy for anomaly list detection with the Landing Gear error is $90.2 \%$, which is considered a very good result. We repeat the same experiment

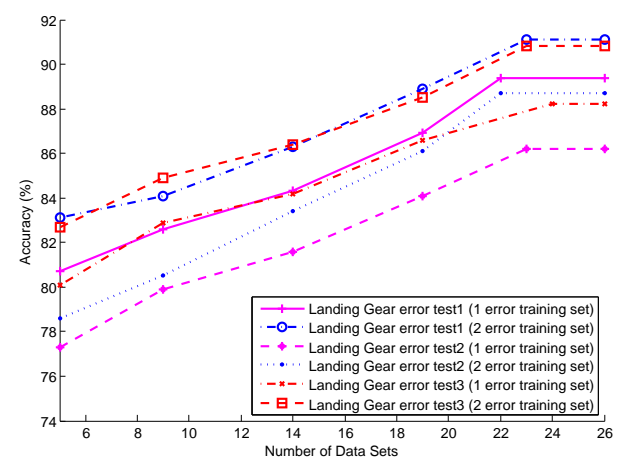

Fig. 3: Landing Gear Error Results

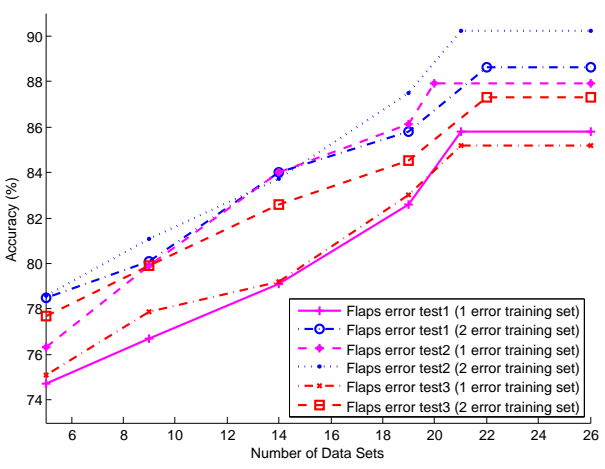

Fig. 4: Flaps Error Results

with the remaining two types of errors separately (Flaps error and Excess Speed error) and we get a combined accuracy results of $88.7 \%$ and $92.3 \%$ respectively. Therefore overall we get a anomaly detection combined accuracy rate of $90.5 \%$ with a confidence range of $\pm 1.8 \%$. 


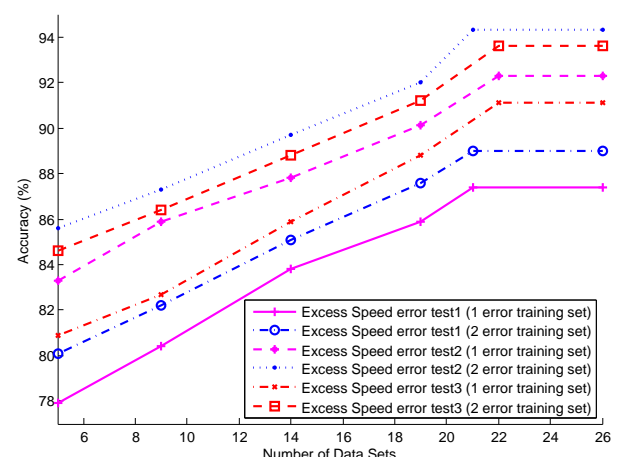

Fig. 5: Excess Speed Error Results

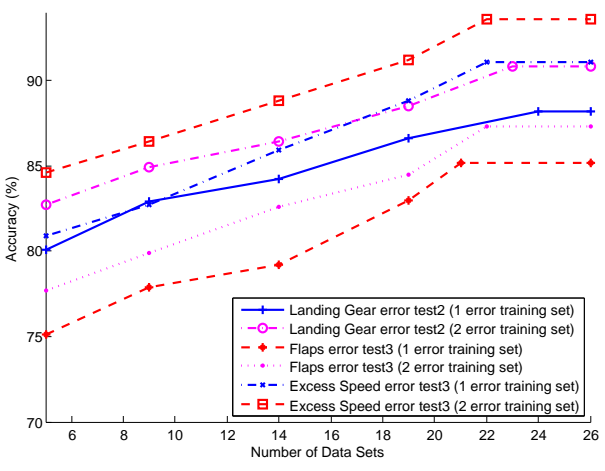

Fig. 6: Error Results Combined

\section{Conclusion}

In this paper we focus on detecting data anomalies in a Dynamic Bayesian Network model, we proposed a novel algorithm to detect data anomalies through backtracking steps of its effect on descendent states until a data anomaly is reached and detected, we have built a DBN model based on pilot actions and instrument data of a flight scenario and then we have implemented our algorithm which has shown robustness in detecting data anomalies that effect other states in the model. Further work can concentrate on the distinction between anomaly types and research its effect on other variables in the model.

\section{References}

1. Hodge, V.J., Austin, J.: A survey of outlier detection methodologies. Artificial Intelligence Review 22 (2004) 85-126

2. Chandola, V., Banerjee, A., Kumar, V.: Anomaly detection: A survey. ACM Computing Surveys 41 (2009) 15:1-15:58

3. Hill, D.J., Minsker, B.S., Amir, E.: Real-time bayesian anomaly detection for environmental sensor data. In: Proceedings of the 32nd conference of the International Association of Hydraulic Engineering and Research, Venice, Italy (July 2007)

4. Shotwell, M.S., Slatey, E.H.: Bayesian outlier detection with dirichlet process mixtures. Bayesian Analysis 4 (2011) 665 - 690

5. Babbar, S., Chawla, S.: On bayesian network and outlier detection. In: Proceedings of the 16th International Conference on Management of Data, Nagpur, India (December 2010)

6. Ferdousi, Z., Maeda, A.: Unsupervised outlier detection in time series data. In: Proceedings of the 22nd International Conference on Data Engineering Workshops, Atlanta, GA, USA (April 2006)

7. Murphy, K.P.: Dynamic Bayesian Networks: Representation, Inference and Learning. PhD thesis, University of California, Berkeley (2002)

8. Pearl, J., Russell, S.: Bayesian networks. In Arbib, M.A., ed.: The Handbook of Brain Theory and Neural Networks. 2nd edn. MIT Press (2003)

9. Hawkins, D.M.: Identification of Outliers. Chapman and Hall (1980) 